\title{
Whither structured representation?
}

Arthur B. Markman

Department of Psychology

University of Texas

Austin, TX 78712

markman@psy.utexas.edu
Eric Dietrich

Program in Philosophy,

Computers, \& Cognitive Science

Binghamton University

Binghamton, NY 13902-6000

dietrich@binghamton.edu

\begin{abstract}
$\underline{\text { Abstract }}$
The perceptual symbol system view assumes that perceptual representations have a role-argument structure. A role-argument structure is often incorporated into amodal symbol systems in order to explain conceptual functions like abstraction and rule-use. The power of perceptual symbol systems to support conceptual functions is likewise rooted in its use of structure. On Barsalou's account, this capacity to use structure (in the form of frames) must be innate.
\end{abstract}

Barsalou's perceptual symbol systems project is exciting and important. Among its virtues are that it lays the groundwork for a more embodied approach to cognition and it subtly changes the way cognitive scientists approach mental content, thereby changing what tools theories of mental content or intentionality can use.

A core distinction in cognitive science is the one between perceptual and conceptual representation. Barsalou points out that this distinction is problematic, because there is no successful demonstration of how these types of representations 
are connected. He suggests that this difficulty should be taken as a sign that perceptual representations are all there is. There are no amodal representations.

In order to make this account plausible, it is critical to demonstrate how key aspects of conceptual representation like abstraction can be cashed out in a perceptual symbol system (PSS). These functions of conceptual representation are accomplished in a PSS by assuming that perceptual representations have roleargument structure in the form of frames.

It is not surprising that Barsalou suggests that frames form the core of PSS. He has long regarded frame representations as being well suited to be the medium underlying conceptual representation (Barsalou, 1992). Further, many cognitive scientists adopted representational systems with some kind of role-argument structure in order to facilitate the abstraction process (see e.g., Fodor, 1981; Markman, 1999; Schank, 1982). And finally, structured representations have been suggested as the basis of models of central perceptual processes like object recognition (Biederman, 1987; Marr, 1982). Thus, there is some reason to believe that perceptual representations are structured, and structured as Barsalou claims they are.

Naturally, the assumption that frames are critical to perceptual symbol systems leads to the question of where the capacity to represent information with frames comes from. There are two possibilities. One is that the frames develop over the life of a cognitive system from simple unstructured representations (e.g., vectors or independent features). A second possibility is that the capacity to build frames is an inherent capability of a PSS. These possibilities are just a version of the standard "learned vs. innate" debate in cognitive science. 
To our knowledge, all attempts to construct complex structured representational schemes starting with only unstructured representations have foundered. For example, attempts to account for the development of complex representational capacities using associative connectionist models were not successful (Fodor \& Pylyshyn, 1988; Marcus, 1998). It is possible to create complex representational structures in a connectionist model, but such structure has to be built in ahead of time using other techniques that give the connectionist system a classical structuring capability (e.g., Shastri \& Ajjanagadde, 1993). A very good example of this is Smolensky's interesting work on connectionism. His tensor product approach does have classical constituent structure already built in (see the series of papers: Smolensky, 1990; Fodor and McLaughlin, 1990; Smolensky, 1995; and McLaughlin, 1997).

In light of this difficulty, Barsalou assumes that the capacity to form structured representations is an inherent component of a PSS. That is, he assumes that stimuli that contact a cognitive agent are converted into frame representations early on, and that the capacity to do this is innate in the system or organism. While structured representations have been incorporated into models of perceptual processes, they are not a necessary component of models of perception (e.g., Ullman, 1996).

The assumption that representations are structured is extraordinarily important for the PSS account. Once these frames are constructed, many of the techniques of concept formation and abstraction used by proponents of structured amodal representations can be incorporated into PSS including the ability to make similarity comparisons and to reason by analogy (Gentner, 1983; Gentner \& Markman, 1997). Thus, once Barsalou assumes that representations in a PSS are 
frames, the ability of this system to account for higher level cognitive abilities is virtually assured.

Where the perceptual symbol system approach differs from previous approaches to structured representations is in assuming that the components of representational frames are tied to perception rather than being derived from a central multimodal representation language (or perhaps from language ability itself). However, it is here that Barsalou's approach has its greatest promissory note. It is a bold step to posit that the structured representations that form the basis of conceptual abilities are closely tied to perception. It is now critical to demonstrate how a true perceptual system could give rise to representations of this type.

\section{References}

Barsalou, L. W. (1992). Frames, concepts and conceptual fields. In A. Lehrer \& E. F. Kittay (Eds.), Frames, Fields and Contrasts: New essays in semantic and lexical organization . Hillsdale, NJ: Lawrence Erlbaum Associates.

Biederman, I. (1987). Recognition-by-components: A theory of human image understanding. Psychological Review, 94(2), 115-147.

Fodor, J. A. (1981). Representations: Philosophical essays on the foundations of cognitive science. Cambridge, MA: The MIT Press.

Fodor, J. and McLaughlin, B. (1990). Connectionism and the problem of systematicity: Why Smolensky's solution doesn't work. Cognition 35 (2), 183-204.

Fodor, J. A., \& Pylyshyn, Z. W. (1988). Connectionism and cognitive architecture: A critical analysis. Cognition, 28, 3-71. 
Gentner, D. (1983). Structure-mapping: A theoretical framework for analogy. Cognitive Science, 7, 155-170.

Gentner, D., \& Markman, A. B. (1997). Structural alignment in analogy and similarity. American Psychologist, 52(1), 45-56.

Marcus, G. F. (1998). Can connectionism save constructivism. Cognition, 66, 153-182.

Markman, A. B. (1999). Knowledge representation. Mahwah, NJ: Lawrence Erlbaum Associates.

Marr, D. (1982). Vision. New York: W.H. Freeman and Company.

McLaughlin, B. (1997). Classical constituents in Smolensky's ICS architecture.

In M. L. Dalla Chiara et al. (eds.) Structures and Norms in Science. 331-343

Schank, R. C. (1982). Dynamic Memory. New York: Cambridge University Press.

Shastri, L., \& Ajjanagadde, V. (1993). From simple associations to systematic reasoning. Behavioral and Brain Sciences, 16(3), 417-494.

Smolensky, P. (1990). Tensor product variable binding and the representation of symbolic structures in connectionist systems. Artificial Intelligence, 46, 159-216.

Smolensky, P. (1995). Reply: constituent structure and explanation in an integrated connectionist/symbolic cognitive architecture. In C. Macdonald and G. Macdonald (eds.) Connectionism: Debates on Psychological Explanation, Oxford: Blackwell. 223-290.

Ullman, S. (1996). High-level Vision. Cambridge, MA: The MIT Press. 